\title{
Total Quality Management (TQM), ein Beitrag zur Sicherung der Wettbewerbsfähigkeit von Unternehmen
}

\author{
Gerd Stegemann
}

\section{Problemstellung}

Qualität hat sich national und international in zurïckliegender Zeit in zunehmendem Maße zu einem der wichtigsten Wettbewerbselemente entwickelt [1]. Verschärfter internationaler Wettbewerb und sich verändernde gesellschaftliche Rahmenbedingungen erfordern von den Unternehmen im Interesse ihrer Zukunftssicherung die Steigerung der Effizienz der unternehmerischen Tätigkeit. Die Überwindung überlebter Führungskonzepte durch die Suche nach und die schrittweise Entwicklung und Realisierung von neuen, für die unternehmensspezifischen Bedingungen adaptierten Managementmethoden ergibt sich daraus als zwingende Notwendigkeit. TQM wird als eine Methode zur Lösung der dargestellten strategischen Herausforderung angesehen.

Nach DIN EN ISO 8402 [2] wird TQM definiert: „Auf die Mitwirkung aller ihrer Mitglieder gestützte Managementmethode einer Organisation, die Qualität in den Mittelpunkt stellt und durch Zufriedenstellung der Kunden auf langfristigen Geschäftserfolg sowie auf Nutzen für die Mitglieder der Organisation und für die Gesellschaft zielt."

Wesentliche Charakteristika des TQM sind demnach:

- das Bestreben nach einer umfassenden Erfüllung der Forderungen externer und interner Kunden mit dem Ziel, einer möglichst dauerhaften Kundenzufriedenheit, wobei der Kundenbegriff die Gesellschaft einschließt,

- die Schaffung von Mitwirkungsmöglichkeiten und deren Realisierung fuir Mitarbeiter aller Stellen und Hierarchieebenen des Unternehmens (Mitarbeiterorientierung) sowie die sich daraus ergebende besondere Verantwortung des Top-Managements sowie

- die Absicht zur Absicherung aller geschäftlichen Ziele (Unternehmensqualität) im Interesse eines langfristigen Geschäftserfolges (letztlich zählt das Ergebnis), auf der Basis einer kontinuierlichen Weiterentwicklung und regelmäßigen Überwachung aller Geschäftsprozesse (Prozeßorientierung).

Unternehmerische Visionen und Leitlinien sind deshalb Basis erfolgreichen TQM's und Ausgangspunkt für unternehmens- und aufgabenspezifische Lösungsansätze.

Unter Berücksichtigung weit verbreiteter Lösungen zum Qualitätsmanagement, z. B. auf Basis zertifizierter QMSysteme nach der ISO 9000-Reihe, können prinzipielle Fragen entstehen, wie:

- Ist TQM eine neue „Wunderwaffe“, der „Königsweg“, die „Patentlösung“ zur Überwindung aller Unter- nehmensprobleme oder nur eine Modeerscheinung (wie andere Managementmethoden vor ihr schon)?

- Erfuillen die in vielen Unternehmen gelebten (und z. B. nach ISO 900X zertifizierten) QM-Systeme ganz oder teilweise die Forderungen von TQM-Konzepten oder handelt es sich bei TQM um eine völlig neue/andere Managementphilosophie?

- Was ist ggf. zu tun, um die im Unternehmen gelebte Qualitätsphilosophie im Sinne zeitgemäßer Unternehmensziele weiter zu entwickeln und umzusetzen?

Es kann sofort festgestellt werden, daß es die „Wunderwaffe“, den „Königsweg“, die „Patentlösung“ für die Erreichung von Spitzenleistungen nicht gibt. Spätestens beim Durchdenken der o. a. globalen Charakteristika von TQM ist festzustellen, daß vieles bereits zu den in den Unternehmen praktizierten strategischen Konzepten gehört und den bisherigen Erfolg dieser ausmacht. Mit einem Zitat von Robert Bosch aus dem Jahre 1919 läßt sich zeigen, daß einzelne Kennzeichen von TQM bereits lange zum Gedankengut deutschen Unternehmertums gehören.

„Es ist mir ein unerträglicher Gedanke, es könnte jemand bei Prüfung meiner Erzeugnisse nachweisen, daß ich irgendwie Minderwertiges leiste. Deshalb habe ich stets versucht, nur Arbeit hinauszugeben, die jeder sachlichen Prüfung standhielt, also sozusagen vom Besten das Beste war."

Andererseits wird bei einem Vergleich von ISO 9000 (steht hier beispielhaft als Systematisierungsgrundlage für die Gestaltung von QM-Systemen) mit Kriterien von TQM-Modellen deutlich, daß neben Gemeinsamkeiten auch qualitative Unterschiede bestehen. Es zeigt sich, daß o. a. Charakteristika des TQM punktuell weit über das Anforderungsprofil der ISO 9000 hinausgehen.

Der Autor teilt jedoch nicht die Auffassung verschiedener Verfasser, wonach wesentliche Kennzeichen von TQM-Modellen (hier gemessen an Selbstbewertungskriterien des EFQM-Modells), z. B. bezüglich des Managements, des Einsatzes und der Erhaltung von Ressourcen, der Kunden- und Mitarbeiterzufriedenheit, der gesellschaftlichen Verantwortung und der Geschäftsergebnisse (insgesamt 59\% des Bewertungsvolumens des EFQM-Modells) auf der Basis der ISO 9000 keinerlei Lösungsansätze bieten (Erfüllungsgrad der TQM-Kriterien gleich $0 \%$ nach [3]).

Umfangreiche Erkenntnisse des Autors und seines Teams bei der Gestaltung von QM-Systemen nach den Leitfäden DIN EN ISO 9004-1 und DIN ISO 9004-2 zeigen sehr wohl große Möglichkeiten zur partiellen Erfuillung des Anforderungsprofils von TQM-Modellen bei Nutzung 
des Interpretationsspielraumes der ISO 9000 -Reihe. So verdeutlichen Ergebnisse aus Untersuchungen des Autors, daß Unternehmen mit entwickeltem QM-System nach der ISO 9000-Reihe durchaus zwischen 35 bis $45 \%$ der durchschnittlichen Punktzahl der Gewinner amerikanischer und europäischer Qualitätsauszeichnugen errreichen.

Der Autor vertritt aus dem Grund die Auffassung, daß das Vorhandensein gelebter QM-Systeme nach der ISO 9000-Reihe (in speziellen Fällen auch anderer Regelwerke) als wichtige Voraussetzung einer erfolgreichen Entwicklung und Implementierung unternehmensspezifischer TQM-Konzepte anzusehen ist (s. Abb. 1).

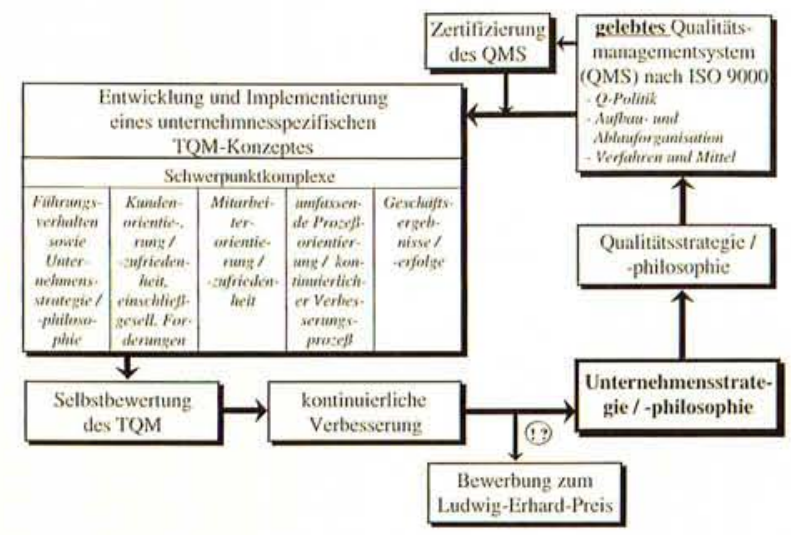

Abb. 1: TQM als Entwicklungsziel und Führungsaufgabe

Die Realisierung betrieblicher TQM-Konzepte wird damit zur „Kür“ auf der Grundlage einer soliden „Pflicht“ (sprich QM-System z. B. nach der ISO 9000-Reihe) zum Qualitätsmanagement.

Es zeigt sich, daß wirtschaftliche und gesellschaftliche Rahmenbedingungen als Stimulator der Entwicklung der Qualitätsarbeit dienen können, ja müssen (Abb. 2).
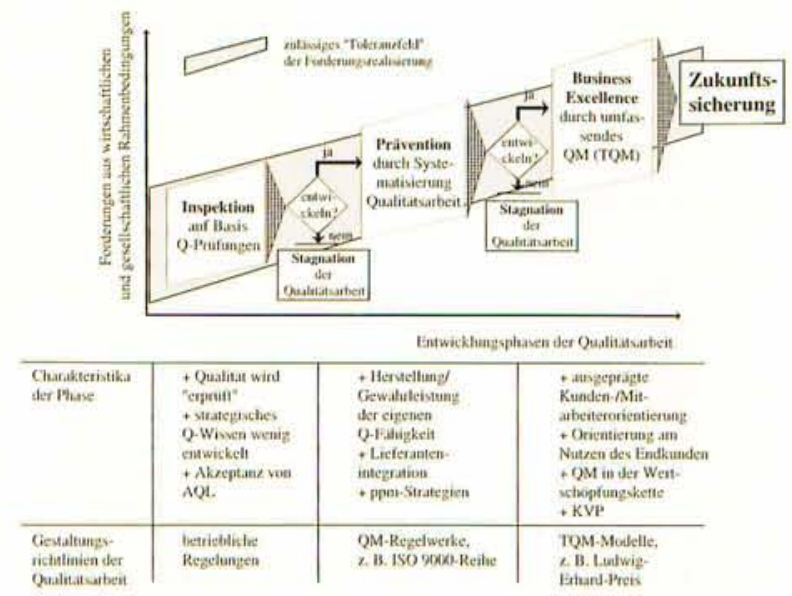

Abb. 2: Charakteristika von Niveaustufen des Qualitätsmanagements [weiterentwickelt nach 4]

Unabhängig davon kann der Weg zum TQM lang und hart, oft aber unverzichtbar sein, verspricht aber ein lohnendes Ziel (s. Tab. 1 und Abb. 3).

So ist nach Angaben von SCHAAR [5] durch die Realisierung von TQM-Konzepten ein Gesamtnutzen von $10 \%$ des Umsatzes realisierbar. Tabelle 1 untersetzt diese Angaben. Die Abhängigkeit des Unternehmenserfolges von der

\begin{tabular}{lcc}
\hline Quelle / Gegenstand & $\begin{array}{c}\text { rel. Nutzen } \\
\%\end{array}$ & Trend \\
\hline Produktivität indirektes Personal & $10-39$ & $\Uparrow$ \\
\hline Produktivität p. a. direktes Personal & 10 & $\Uparrow$ \\
\hline Reduzierung Bestände & $25-50$ & $\Downarrow$ \\
\hline Reduzierung Durchlaufzeiten & $25-90$ & $\Downarrow$ \\
\hline Beschleunigung Entwicklungszeiten & $50 \%$ & $\Uparrow$ \\
\hline Reduzierung Produktfehler & $50-99$ & $\Downarrow$ \\
\hline Reduzierung manueller Arbeitsfehler & 80 & $\Downarrow$ \\
\hline Termintreue in allen Funktionen & 90 & $\Uparrow$ \\
\hline
\end{tabular}

Tab. 1: Einsparungspotentiale durch TQM [5]

Ausprägung ihrer Qualitätsfähigkeit wird in [9] am Beispiel der Umsatzrendite und des Umsatzwachstums quantifiziert (Abb. 3).

Unternehmen ohne klare Systematisierung ihrer qualitätsrelevanten Tätigkeiten (z. B. durch ein QM-System) oder mit Problemen bei der Einrichtung/Erhaltung eines normenkonformen QM-Systems werden nach den Erkenntnissen des Autors geringe Chancen für die erfolgreiche Entwicklung eines TQM-Konzeptes haben.
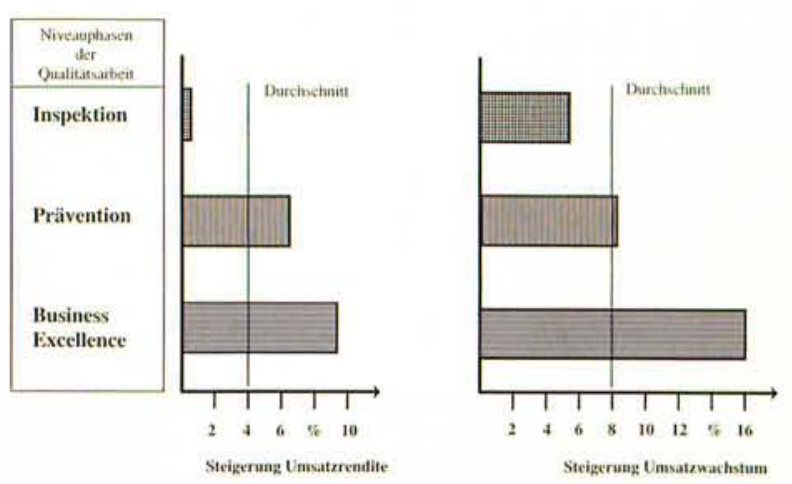

Abb. 3: Unternehmenserfolg in Abhängigkeit ihrer Qualitätsfähigkeit [9]

Die Realisierung von TQM-Konzepten stellt also eine große Anstrengung für jedes Unternehmen dar (Visionen entwickeln) und setzt eine schonungslose Analyse betrieblicher Bedingungen sowie die Bereitschaft zu tiefgreifenden Veränderungen voraus (eingefahrene Wege verlassen).

\section{TQM-Modelle}

Nach Auffassung des Autors stellt sich nicht die Frage, ob im Unternehmen TQM-Aspekte Gegenstand der unternehmerischen Tätigkeit sind oder werden, sondern wann und in welchem Umfang sie zu realisieren sind. Zukunftssicherung zum Nutzen der Mitglieder der Organisation und der Gesellschaft durch Zufriedenstellung (Begeisterung) des Kunden wird als wichtigstes Entscheidungskriterium zum „Wann?“ und „In welchem Umfang?“ des TQM's angesehen.

In diesem Prozeß wird in Deutschland der Ludwig-Erhard-Preis eine wichtige Rolle spielen. Das Bewertungsmodell des Ludwig-Erhard-Preises (Abb. 4) schließt die 
Mittel und Wege sowie die Ergebnisse unternehmerischer Tätigkeit im Sinne des TQM zu je der Hälfte des Bewertungsvolumens von insgesamt 1000 Punkten ein. Eine Fokussierung der Kriterien dieses Bewertungsmodelles auf die Rolle des Managements bei der Entwicklung einer hohen Kunden-, Mitarbeiter- und Prozeßorientierung wird genauso deutlich, wie die Bewertung des Qualitätsmanagements an den wirtschaftlichen Ergebnissen des Unternehmens unter Berücksichtigung berechtigter gesellschaftlicher Interessen.

Es wird davon ausgegangen, daß der Nutzen aus der „Beschäftigung“ mit dem Ludwig-Erhard-Preis für den überwiegenden Teil der Unternehmen in den notwendigen Stärke-Schwäche-Analysen basierend auf Selbstbewertungen sowie dem Vergleich mit anderen Unternehmen (externes Benchmarking) und den sich daraus ergebenden Möglichkeiten für kontinuierliche Verbesserungen ergibt. Er fördert damit die Auseinandersetzung mit den Ideen des TQM.

Insbesondere die tendenzielle Weiterentwicklung zum umfassenden Qualitätsmanagement (TQM) führte dazu, daß amerikanische Automobilhersteller (Chrysler, Ford, GM) im Jahre 1994 ein Regelwerk mit erhöhten Forderungen im Vergleich mit der ISO 9001 für die branchenspezifische Zertifizierung in Kraft setzten (QS 9000). Vergleichbare Regelwerke existieren auch für die Automobilbranche in Europa (z. B. in Deutschland, Frankreich, Italien). In Deutschland betrifft das die Weiterentwicklung von VDA 6 zu VDA 6.1 (QM-Systemaudit - für materielle Produkte) aus dem Jahre 1996 sowie den Band 6, Teil 2 (QM-Systemaudit für immaterielle Produkte).

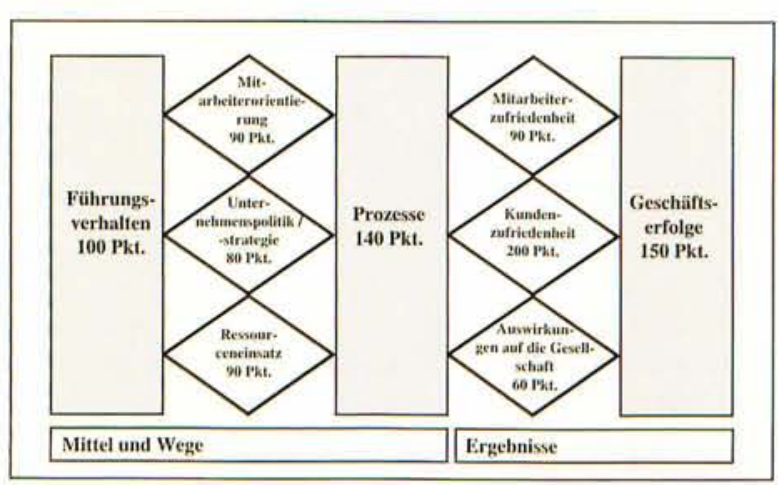

Abb. 4: Bewertungsmodell des Ludwig-Erhard-Preises

Formal erstreckt sich der Geltungsbereich dieser Regelwerke auf die Automobilindustrie und ihre Partner. Ihre Orientierung am TQM sowie die Stellung der Automobilindustrie in den Volkswirtschaften lassen erwarten, daß die verallgemeinerungsfähigen Aspekte (Abb. 5) entweder direkt im Qualitätsmanagement anderer Branchen oder indirekt über den Einfluß dieser Regelwerke auf die Weiterentwicklung der ISO 9000-Reihe zum Tragen kommen werden.

Trotz des orientierenden Charakters dieser Standards darf nicht übersehen werden, daß das Vorhandensein unterschiedlicher Forderungskataloge zum Qualitätsmanagement im konkreten Fall die Weiterentwicklung

\begin{tabular}{|c|}
\hline $\begin{array}{c}\text { Übertragbare Forderungen für } \\
\text { andere Branchen }\end{array}$ \\
\hline 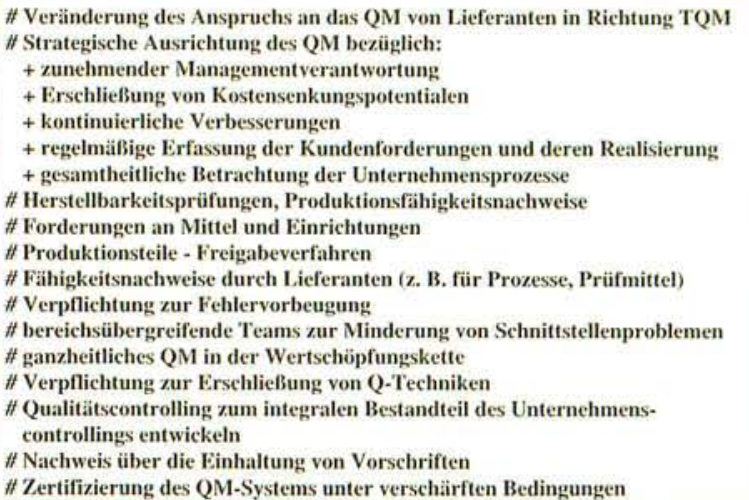 \\
\hline
\end{tabular}

Abb. 5: Verallgemeinerungsfähige Forderungen aus QS 9000 und VDA 6.1/ 6.2 für die strategische Weiterentwicklung des Qualitätsmanagements

vorhandener QM-Systeme (nach der ISO 9000-Reihe), insbesondere für kleine und mittlere Unternehmen erschwert. Der einzig gangbare Weg für die Gestaltung anforderungsgerechter QM-Systeme besteht nach Auffassung des Autors für diese Unternehmensgruppen in der Harmonisierung der verschiedenen Forderungsprofile und die Umsetzung sich daraus ergebender Gestaltungsforderungen in den betrieblichen QM-Systemen (Abb. 6).

Im Beispiel wird von der Existenz eines betrieblichen QM-Systems auf der Basis der ISO 9000-Reihe und einer angestrebten Harmonisierung der Forderungen aus den Regelwerken QS 9000 und VDA 6.1 ausgegangen.

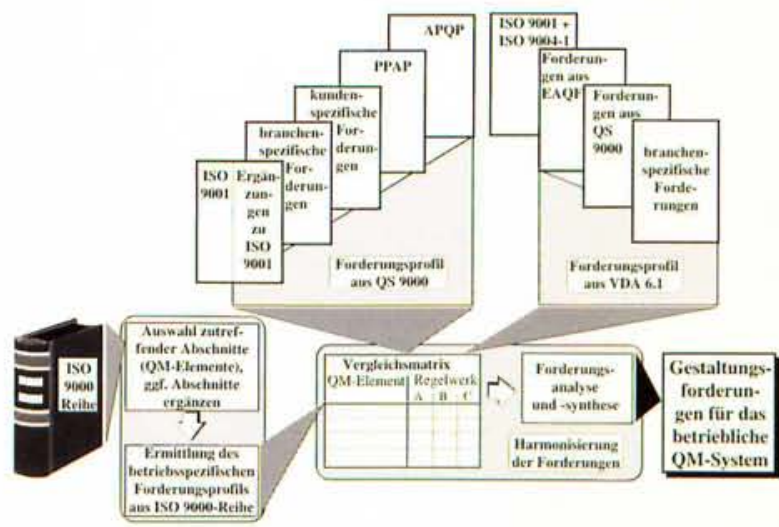

Abb. 6: Weg für die Ermittlung von Gestaltungsforderungen für das betriebliche QM-System

\section{Beispielhafte Lösungsansätze zur Ent- wicklung des TQM im Unternehmen}

\subsection{Entwicklung des TQM als Führungsaufgabe}

Die Entscheidung zur Entwicklung des TQM muß von der Unternehmensführung ausgehen und vom Top-Management getragen werden.

Es spricht nicht für deutsche Manager, wenn in [10] dargestellt ist, daß $67 \%$ der Mitarbeiter Akzeptanzprobleme zum Qualitätsmanagement haben.

Nach von DIEMER [8] erfordert der schnelle Wandel von Menschen und Organisationen ein hohes Maß an dyna- 
mischem Verhalten. Der sich dabei einstellende unterschiedliche Reifegrad ist vor allem durch das Verhalten, ob die Energie vornehmlich für die Abwehr von Neuerungen oder für deren Umsetzung genutzt wird, zu charakterisieren [8]. In Verallgemeinerung dieser Aussage richtet sich Mitarbeiterorientierung deshalb im Schwerpunkt auf die Erschließung des Potentials der Mitarbeiter im Sinne der beschleunigten Umsetzung des Neuen. Dazu sind durch die Führungstätigkeit Voraussetzungen für ein selbständiges Handeln der Mitarbeiter zu schaffen (Befähigung durch Weiterbildung, Schaffung organisatorischer und materieller Voraussetzungen, z. B. Teamarbeit, Arbeitsbedingungen, Arbeitssicherheit). Eine frühzeitige Einbeziehung der Mitarbeiter in den Innovationsprozeß zur Entwicklung des TQM baut Hemmschwellen ab, erhöht die Akzeptanz für diesen Prozeß und erschließt frühzeitig das Know-how der Mitarbeiter. Der Autor hält es für besonders wichtig, den Mitarbeitern Möglichkeiten für eine interdisziplinäre Zusammenarbeit (auch über das Unternehmen hinaus, z. B. in der externen Wertschöpfungskette) zu schaffen. Gezieltes Projektmanagement sichert dabei eine effektive Projektrealisierung im Sinne der Unternehmensziele und bildet eine gute Basis furr eine zielorientierte Kommunikation zwischen Mitarbeitern und Organisation. Auf der Basis der „Visionen“ der Unternehmensführung ist das Feedback möglichst vieler Mitarbeiter der Organisation sowie von Partnern aus der Wertschöpfungskette zu „organisieren“ (s. Abschnitt 3.2) (s. Abb. 7).

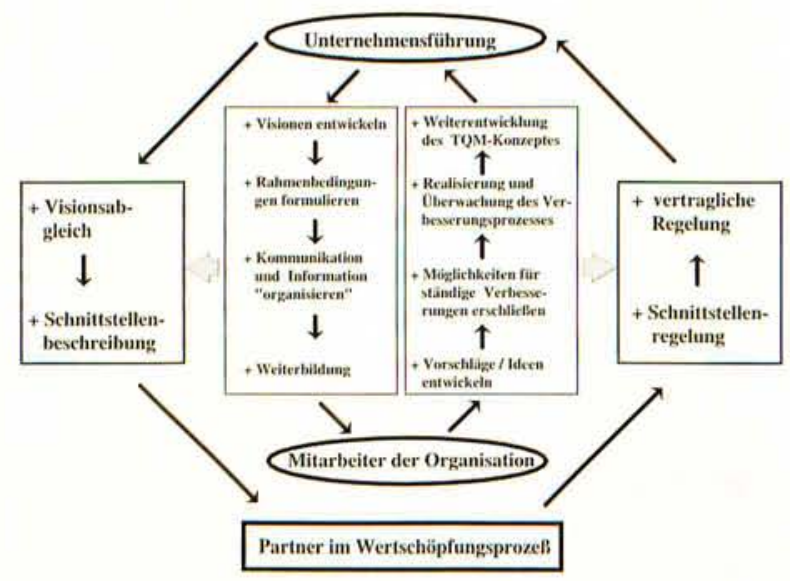

Abb. 7: Wechselverhältnis der Führungstätigkeit zum TQM

Der bei der Einrichtung eines QM-Systems nach der ISO 9000-Reihe vielfach beobachtete Hang zur „Delegierung“ von „strategischen Aufgaben“, z. B. an den Qualitätsbeauftragten ist bei der Entwicklung des TQM so nicht möglich.

Unabdingbare „Start-Aufgabe“ der Unternehmensführung ist die Ableitung und Kommunizierung der Qualitätsphilosophie/-leitlinien auf der Basis der Unternehmensstrategie/-philosophie (s. Abb. 1). Sie bildet die Grundlage für die Beschreibung von Unternehmenszielen. Von großer Bedeutung ist eine langfristige Anlage der so entstehenden Visionen und die frühzeitige Einbeziehung der Mitarbeiter in die Innovationsphase (Betriebsrat nicht vergessen!) (Kommunikations- und Infor- mationspflicht). Kontinuität der Entwicklung ist grundsätzlich anzustreben, Diskontinuitäten sind jedoch nicht vermeidbar, weil teilweise unverzichtbar:

Es sei an dieser Stelle darauf hingewiesen, daß die verschiedenen Regelwerke spezielle Forderungen zur Dokumentation des QM beinhalten. Hier ergibt sich ein großes Feld in der Führungstätigkeit. Die in größeren Unternehmen anzutreffende Praxis, parallel QM-Dokumentationen entsprechend den Forderungen verschiedener Regelwerke zu haben, erscheint dem Autor für kleine und mittlere Unternehmen praktisch nicht relevant. Der Autor geht davon aus, daß das Dokumentationskonzept zur Entwicklung des TQM aufbauend auf der vorhandenen QM-Dokumentation des QM-Systems nach ISO 9000 abzuleiten ist. Die Dokumentation zum QM-System nach ISO 9000 bildet danach das „Fundament" der Dokumentation zum TQM.

Gute Voraussetzungen für die Realisierung dieser Prozesse haben Unternehmen, in denen bei Einrichtung und Erhaltung eines QM-Systems nach der ISO 9000Reihe die Forderungen zu den Führungselementen (z. B. zur Qualitätspolitik sowie zu -zielen) nicht nur formal „behandelt" wurden und in denen ein gezieltes Projektmanagement selbstverständlich ist.

Zu den Kernaufgaben der Führungstätigkeit bei der Entwicklung des TQM im Unternehmen ist die Planung, Organisation, Realisierung und Überwachung der Verbesserungsprozesse zu rechnen. Interne Audits, Selbstbewertungen, Qualitätscontrolling, Benchmarking sind Werkzeuge, die diesen Prozeß unterstützen können. Insbesondere Benchmarking bietet Möglichkeiten zur Erschließung überbetrieblichen Wissens.

In [7] wird darauf verwiesen, daß die Verbesserungsbemühungen neben den traditionellen Gegenständen, wie Fertigungs- oder Dienstleistungsablauf stärker bisher vernachlässigte Themen, wie Arbeitsorganisation und Kommunikation berüicksichtigen sollten.

Von sehr großer allgemeiner Bedeutung für eine planmäßige Entwicklung des TQM's im Unternehmen wird die Gestaltung des Qualitätscontrollings angesehen. Nach Auffassung des Autors ist unbedingt auf eine konsequente Integration des Qualitätscontrollings in das Unternehmenscontrolling zu achten. Dabei wird Qualitätscontrolling als Methode zur Befähigung des Managements auf der Grundlage von Abweichungsanalysen die Festlegung und Verfolgung von Qualitätszielen im Sinne der Qualitätslenkung angesehen. Bei der Entwicklung entsprechender (branchenspezifischer) Kennzahlensysteme besteht international großer Entwicklungsbedarf. Die zu entwickelnden Kennzahlensysteme sind an meßbaren Effiziensindikatoren auszurichten. Wobei Effizienz letztlich daran zu beurteilen ist, wie und mit welchem Aufwand es gelingt, die Übereinstimmung zwischen Kundenforderungen und Nutzen für die Organisation und Gesellschaft herzustellen. Zu überwinden ist die derzeitig häufig vorzufindende Praxis der inhaltlichen Trennung von Kennzahlen zur Qualitätsbewertung und solchen für übrige betriebliche Prozesse. 
Das Streben nach höchstmöglicher Kundenzufriedenheit muß Kernpunkt der Entwicklung des TQM's sein und erfordert sicher in allen Unternehmen zusätzliche Anstrengungen.

TQM stellt die Wahrnehmungen des Kunden bezüglich der Leistungen des Lieferanten in den Vordergrund, macht diese Wahrnehmungen zum Bewertungsmaßstab.

Dabei ist die überbetriebliche Ausrichtung (in der gesamten Wertschöpfungskette, s. Abschnitt 3.2) an den Forderungen des Endkunden (was z. B. auch die Akzeptanz eines erforderlichen Kundennutzens bei betriebswirtschaftlichen Überlegungen des Lieferanten) besonders hervorzuheben.

An Ergebnissen aus Untersuchungen zur Entwicklung des Qualitätsmanagements im Land Brandenburg läßt sich zeigen, daß bezüglich der Entwicklung des Führungsverhaltens zum TQM, dargestellt an Beispielen zur Kunden-, Mitarbeiter- und Prozeßorientierung große Entwicklungspotentiale bestehen [11].

- Beispiel zur Kundenorientierung [11]: Obwohl eine hohe Kundenzufriedenheit als wichtiges strategisches Ziel der Qualitätsarbeit angestrebt wird (s. Abb. 8) und die Kunden als wichtige Partner bezüglich der Gestaltung des Qualitätsmanagements angsehen werden (75 bis $89 \%$ der Unternehmen sind dieser Auffassung), spielt die quantifizierbare Bewertung der Dienstleistungen, Produkte und Kundenbeziehungen durch die Kunden selber eine untergeordnete Rolle (Tab. 2).

- Beispiel zur_Mitarbeiterorientierung [11]: $79 \%$ der befragten Unternehmen bewerten in verschiedenster Form die Zufriedenheit ihrer Mitarbeiter.
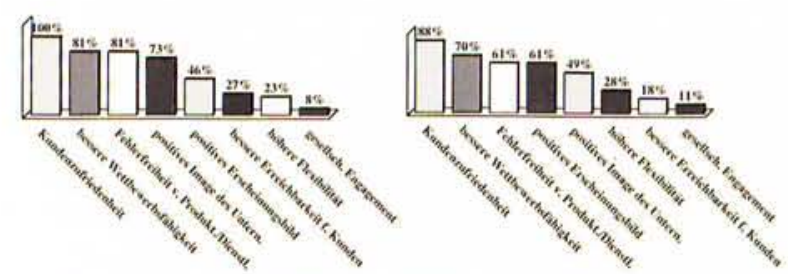

a) Unternehmen mit QM-System

b) Unternehmen ohne QM-System

Abb. 8: Strategische Ziele der Qualitätsarbeit im Unternehmen [11]

\begin{tabular}{|l|c|}
\hline Kriterium der Bewertung & $\begin{array}{r}\text { Anteil der Unterneh- } \\
\text { men, die Kriterium } \\
\text { bewerten lassen [\%] }\end{array}$ \\
\hline Qualitätsfähigkeit des Unternehmens & 21 \\
\hline Termintreue & 15 \\
\hline Reklamationsabwicklung & 12 \\
\hline $\begin{array}{l}\text { Flexibilität des Unternehmens auf } \\
\text { Kundenforderungen }\end{array}$ & 9 \\
\hline Kundenservice & 6 \\
\hline Preis & 3 \\
\hline
\end{tabular}

Tab. 2: Angaben zur Bewertung ausgewählter Kriterien des Qualitätsmanagements auf der Grundlage schriftlicher Kundenbefragungen |11|
Bezogen auf alle befragten Unternehmen führen jedoch nur $15 \%$ der Unternehmen schriftliche Mitarbeiterbefragungen durch (s. Abb. 9), alle anderen Bewertungen entfallen auf solche zwar wichtigen aber häufig sehr allgemeinen Bewertungsformen, wie Ergebnisse aus Mitarbeiterversammlungen oder Mitarbeitergesprächen.

Geht man nach Abb. 9, so stellen nur $4 \%$ der Unternehmen durch Mitarbeiterbefragungen den Kundenbezug her (jeweils $4 \%$ fordern die Auffassung ihrer Mitarbeiter zur Kundenzufriedenheit bzw. zu Kundenforderungen ein).

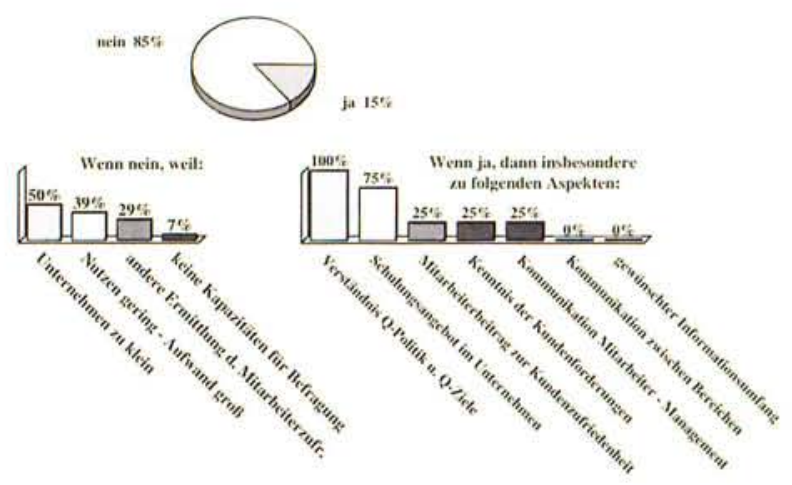

Abb. 9: Umfang schriftlicher Mitarbeiterbefragungen sowie Pro- und KontraArgumente [11]

Große Potentiale bieten sich für eine verstärkte Einbeziehung von Mitarbeiter (s. Tab. 3).

\begin{tabular}{|l|c|c|}
\hline \multirow{2}{*}{$\begin{array}{l}\text { Form der } \\
\text { Mitarbeitereinbeziehung }\end{array}$} & \multicolumn{2}{|c|}{ Unternehmen } \\
\cline { 2 - 3 } & $\begin{array}{c}\text { mit } \\
\text { QM-System }\end{array}$ & $\begin{array}{c}\text { ohne } \\
\text { QM-System }\end{array}$ \\
\hline $\begin{array}{l}\text { Verbesserungsvorschlags- } \\
\text { wesen }\end{array}$ & $15 \%$ & $26 \%$ \\
\hline $\begin{array}{l}\text { Problemlösungsgruppen- } \\
\text { arbeit }\end{array}$ & $15 \%$ & $6 \%$ \\
\hline Gruppenarbeit & $12 \%$ & $9 \%$ \\
\hline
\end{tabular}

Tab. 3: Nutzung ausgewählter Formen der Mitarbeitereinbeziehung in die Qualitätsarbeit [11]

- Beispiel zur_Prozeßorientierung [11]: $96 \%$ der befragten Unternehmen geben an, regelmäßig die Wirksamkeit von Geschäftsprozessen zu bewerten (Abb. 10).

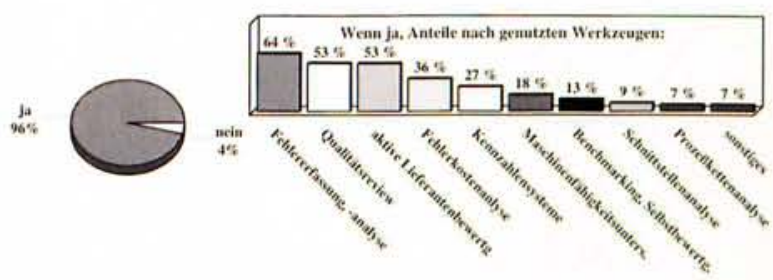

Abb. 10: Anteil der Unternehmen, die Unternehmensprozesse bezüglich ihrer Wirksamkeit bewerten und Anteile der dazu genutzten Werkzeuge [11]

Unbefriedigend ist, daß Werkzeuge, wie Maschinenfähigkeitsuntersuchungen (18\%), Benchmarking (13\%) oder Prozeßkettenanalysen (7\%) dabei eine sehr untergeordnete Rolle spielen. 


\subsection{TQM in der externen Wertschöpfungskette}

Die Herstellung der Kundenzufriedenheit erfordert neben den unternehmerischen Aktivitäten im eigenen Unternehmen ein partnerschaftliches Zusammenwirken in der Wertschöpfungskette (Wertschöpfungskette im Sinne von [6] gebraucht) (Abb. 11).

In [6] wird das partnerschaftliche Zusammenwirken wie folgt beschrieben:

„Eine Partnerschaft schafft und erhält Wettbewerbsvorteile der beteiligten Parteien gegenüber ähnlichen Organisationen durch das Zusammenführen von Ressourcen in einer auf kontinuierliche gegenseitige Verbesserungen konzentrierten Atmosphäre des Vertrauens."

Als wesentlicher Erfolgsfaktor wird dazu ein auf die Wünsche/Erfordernisse des Endkunden ausgerichtetes und durchgängiges Qualitätsmanagement angesehen.
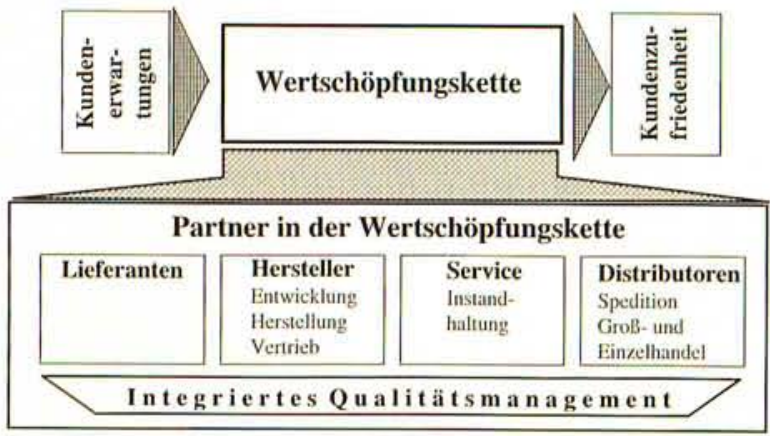

Abb. 11: Partner in der Wertschöpfungskette

Diese durchgängige Partnerschaft zu entwickeln und zu realisieren ist unter praktischen Bedingungen gewöhnlich nicht einfach (z. B. wenn auch internationale Partner zu berücksichtigen sind). Partiell besteht sie jedoch (z. B. in Kooperationen) und wird auch gefordert, z. B. im Rahmen der Beschaffung (s. DIN EN ISO 9004-1, Element 9.1 oder VDA 6.1, Abschnitt 11.2 oder QS 9000, Element 4.6.2) und von den Unternehmen erkannt und realisiert (Abb. 12).

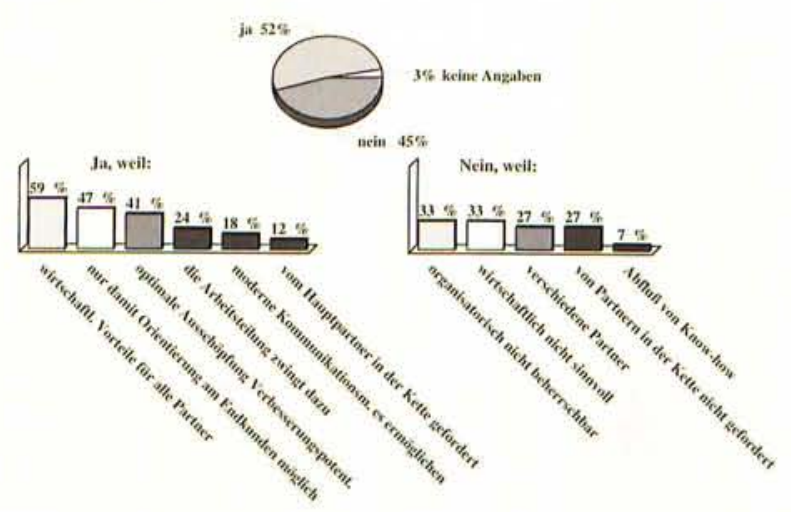

Abb. 12: Einbindung des unternehmensinternen Qualitätsmanagements innerhalb der externen Wertschöpfungskette [11]

Bei der Entwicklung des TQM kommt es unabhängig davon sicherlich vielerorts deshalb zunächst darauf an, die „geistige Bereitschaft“ zur partnerschaftlichen $\mathrm{Zu}$ sammenarbeit herzustellen. Für das eigene Unternehmen sind dazu Bedingungen zu schaffen, die eine derartige Zusammenarbeit ermöglichen und befördern (Abb. 13).

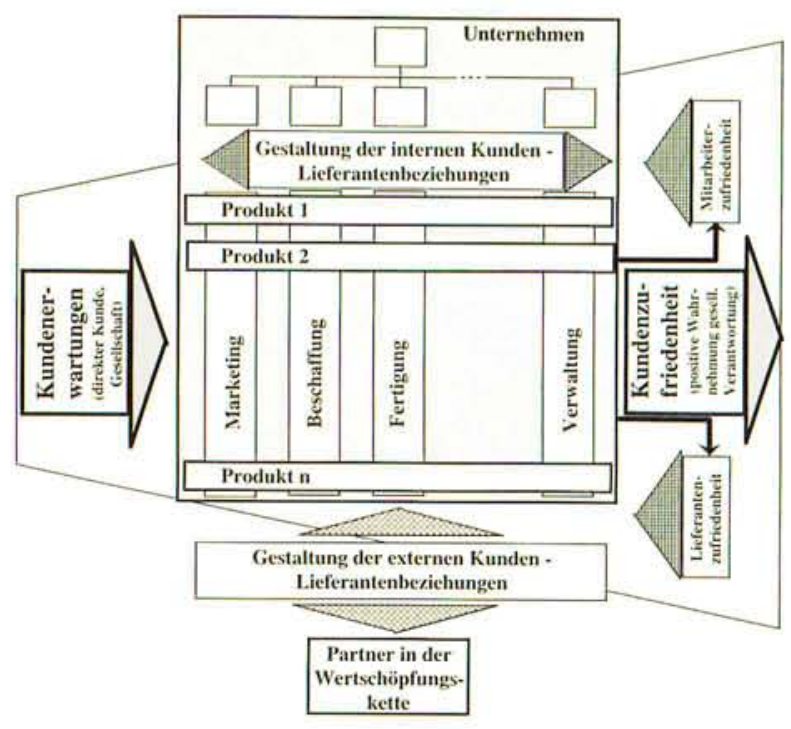

Abb. 13: Beziehungsstruktur zwischen Unternehmensorganisation und Produkt im Spannungsfeld zwischen Kundenerwartungen und-zufriedenheit

Grundsätzlich läuft dies auf die Gestaltung der internen und externen Kunden-Lieferantenbeziehungen hinaus. Im Unternehmen sind flache Organisationsstruktur, ganzheitlich realisierbare Arbeitsaufgaben horizontal in der Organisation (produktbezogene Gruppenarbeit) mit Möglichkeiten zur Selbstabstimmung sowie Kommunikations- und Informationspflicht Voraussetzungen dafür. Im Sinne der Erfuillung von Kundenforderungen werden Schnittstellenanalysen, -beschreibungen und -regelungen als Ausgangspunkt zur Gestaltung der externen Kunden-Lieferantenbeziehungen angesehen.

\section{Stufenmodell zur Entwicklung des TQM}

Es wird deutlich, daß die schrittweise Entwicklung des TQM eine unabdingbare Aufgabe der Zukunftssicherung für jedes Unternehmen wird. Das „Wann?“, „Wie?“, „In welchem Umfang?" sind unternehmensspezifisch zu entscheiden. Durch die permanente Entwicklung der Forderungen an die Unternehmensstrategie/-philosophie gibt es keinen „Endpunkt“ des TQM. Der Autor gebraucht deshalb nicht den Begriff „Einführung des TQM“ (Einführung suggeriert einen endlichen Prozeß). Vorliegende TQM-Modelle (z. B. Ludwig-Erhard-Preis) oder Regelwerke (z. B. QS 9000 oder VDA 6.1 bzw. 6.2) sind einerseits Ergebnis dieser notwendigen Entwicklung und andererseits wichtige Werkzeuge für die Entwicklung des TQM im Unternehmen. Ein Vergleich des Forderungsprofils herkömmlicher Modelle zur Gestaltung des Qualitätsmanagements mit o. a. TQM-Modellen offenbart notwendige qualitative Veränderungen der Qualitätsarbeit, insbesondere abgeleitet aus dem eigentlichen Sinn jeglicher unternehmerischen Tätigkeit, auf der Grundlage der Zufriedenstellung der (End-)Kunden, den Geschäftserfolg langfristig zu sichern. Von großer Bedeutung ist dabei die Einbeziehung der „Gesellschaft" in den Kundenbegriff.

Dadurch bedingt entstehen neue Ansprüche an die unternehmerische Führungstätigkeit. Ungeachtet dessen zeigt der o. a. Vergleich, daß TQM nichts völlig Neues 
ist, sondern wichtige Charakteristika des TQM schon lange zu den Prinzipien erfolgreicher, unternehmerischer Tätigkeit gehören. Insbesondere das Vorhandensein eines gelebten QM-Systems (z. B. nach ISO 9000) kann als gute (der Verfasser ist der Auffassung als notwendige) Voraussetzung zur Entwicklung des TQM's im Unternehmen angesehen werden. Nachfolgende Betrachtungen zu Schritten der Entwicklung des TQM's (Stufenplan - Abb. 14) gehen von dieser Bedingung aus. Als Voraussetzung für eine erfolgreiche Entwicklung des TQM wird eine schonungslose Analyse durch das Management zum Stand der Qualitätsarbeit im Unternehmen sowie zu Anlässen, Zielen und Aufwendungen für die Weiterentwicklung des Qualitätsmanagements im Unternehmen angesehen. Auf der Grundlage klarer Vorstellungen zum anzuwendenden TQM-Modell werden Führungsgrundsätze und Rahmenbedingungen zur Entwicklung des TQM formuliert (s. Abb. 7).

Diese Maßnahmen sind grundsätzlich mit der Schulung des Managements zu verbinden.

Die eigentliche Bewertung der qualitätsrelevanten Tätigkeiten erfolgt mit Hilfe eines Analyseaudits/Assessments nach einer um den TQM-Bezug erweiterten ISOCheckliste. Die Ergebnisse dieser Bewertung bilden die Grundlage für die Erarbeitung des Stufenplanes und des Dokumentationskonzeptes, wobei bei Letzterem unbedingt von einer Weiterentwicklung der vorliegenden QM-Dokumentation ausgegangen wird. Entscheidend für eine erfolgreiche Entwicklung des TQM im Unternehmen ist ein zielstrebiges Projektmanagement.

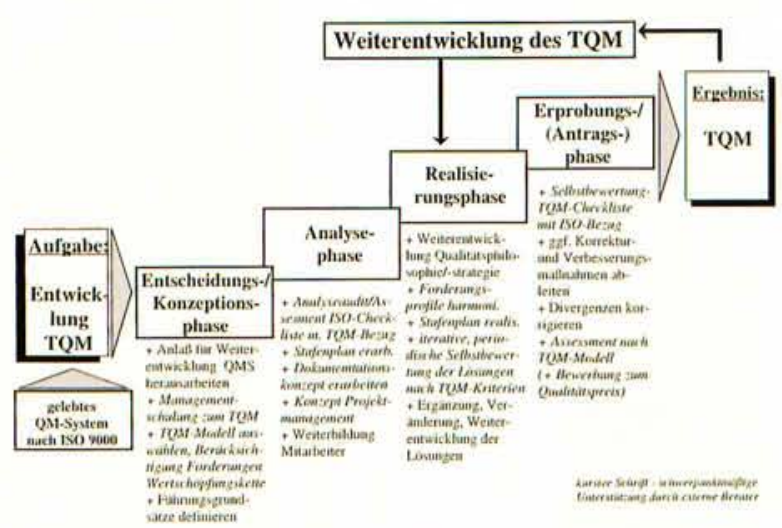

Abb. 14 Stufenplan zur Entwicklung des TQM

Es wird davon ausgegangen, daß Führungskräfte genauso in das Projektteam einbezogen werden, wie externe Berater. Zu den ersten Aufgaben des Projektteams gehört eine aufgabenbezogene Weiterbildung der Mitarbeiter (permanenter Prozeß). Hier ist der Ausgangspunkt für die Weiterentwicklung der Qualitätsphilosophie/-strategie des Unternehmens. Wichtig ist, daß auf dieser Grundlage sofort Unternehmensziele und aus diesen Maßnahmen für den Verbesserungsprozeß abgeleitet werden.

Eine unabdingbare (u. U. sehr komplizierte) Aufgabe ist die erforderliche Harmonisierung der Forderungsprofile ggf. verschiedener Regelwerke. Die sich anschließende eigentliche Realisierung des Stufenplanes erfolgt nach vorzugebenden Prioritäten. In den Realisierungsprozeß werden periodisch Selbstbewertungen eingeordnet. Sie bilden die Basis für ggf. erforderliche Ergänzungen, Veränderungen oder Weiterentwicklungen der Lösungen. Der Implementierung des TQM sollte eine umfassende Erprobung mit dem Bestreben ggf. vorhandene Divergenzen zu korrigieren folgen.

\section{Zusammenfassung}

Die Entwicklung des TQM im Unternehmen ist eine wichtige Methode zur Gewährleistung der Zukunftssicherung. Zeitpunkt und Umfang der Entwicklung des TQM sind unternehmensspezifisch festzulegen und $\mathrm{zu}$ realisieren. Gute Voraussetzungen für die Entwicklung des TQM bietet das gelebte QM-System des Unternehmens. Der Entwicklungsprozeß des TQM ist langfristig anzulegen und bedarf in vielen kleinen und mittleren Unternehmen einer Begleitung durch ausgewiesene externe Berater.

\section{Literatur}

(1) Stegemann, G., Qualitätsmanagement - Chance und Herausforderung für kleine und mittelständische Unternehmen. In: Tagungsmaterial 4. Wildauer Workshop Qualitätsmanagement vom 19.12.1997

[2] DIN EN ISO 8402 Qualitätsmanagement Begriffe, Ausgabe August 1995

[3] Zink. K. J., TQM als integratives Managementkonzept. Carl Hanser Verlag München Wien 1995, S. 42 und 43

[4] Malorny, C., TQM umsetzen - Der Weg zu Business Excellence. Schäffer-Poeschel Verlag Stuttgart 1996, S. 330

[5] Schaar, H., Die ganzheitliche Unternehmensentwicklung unter den Aspekten des TQM. In: Die hohe Schule des Total Quality Management. Springer Verlag Berlin u. a. 1994, S. 78

[6] Poirier, C. C./Reiter, S. E., Die optimale Wertschöpfungskette. Campus Verlag Frankfurt/New York 1997

[7] Moldaschl, M., KVP als Kooperationsverhinderungsprogramm. In: Qualität und Zuverlässigkeit 42 (1997) 4 , S. 404

[8] von Diemer, R., Das TQM-Verhalten ändern. In: Qualität und Zuverlässigkeit 42 (1997) 4, S. 390

[9] McKinsey \& Company, Mit Hochleistungskultur und Kundennutzen an die Weltspitze - Qualität gewinnt. Schäffer-Porschel Verlag Stuttgart 1995

[10] Hohe Bewertung des Qualitätsmanagements durch Topmanager. In: Qualität und Zuverlässigkeit 41 (1996) 12 , S. 1351

[11] Stegemann, G./Kauschke, S./Krüger, R., Erarbeitung eines Konzeptes für die wissenschaftliche Begleitung zum Operationellen Programm, Förderschwerpunkt Qualitätsmanagement, im Land Brandenburg. Technische Fachhochschule Wildau und Verein für Qualitätsförderung Brandenburg, 1998

\section{Verfasser}

Prof. Dr.-Ing. habil. Gerd Stegemann

Technische Fachhochschule Wildau

Fachbereich Ingenieurwesen/Wirtschaftsingenieurwesen Tel. (0 33 75) 508-914 